TRANSACTIONS OF THE

AMERICAN MATHEMATICAL SOCIETY

Volume 300, Number 2, April 1987

\title{
A GENERAL THEORY OF CANONICAL FORMS
}

\author{
RICHARD S. PALAIS AND CHUU-LIAN TERNG
}

\begin{abstract}
If $G$ is a compact Lie group and $M$ a Riemannian $G$-manifold with principal orbits of codimension $k$ then a section or canonical form for $M$ is a closed, smooth $k$-dimensional submanifold of $M$ which meets all orbits of $M$ orthogonally. We discuss some of the remarkable properties of $G$-manifolds that admit sections, develop methods for constructing sections, and consider several applications.
\end{abstract}

0. Introduction. Let $G$ be a compact Lie group acting isometrically on a Riemannian manifold $M$. Then the image $S$ of a small ball in the normal plane $\nu(G x)_{x}$ under the exponential map is a smooth, local $G_{x}$-slice, which in general cannot be extended to a global slice for $M$. A section $\Sigma$ for $M$ is defined to be a closed, smooth submanifold of $M$ which meets every orbit of $M$ orthogonally. A good example to keep in mind is perhaps the most important of all canonical form theorems; namely for $M$ we take the Euclidean space of symmetric $k \times k$ matrices with inner product $\langle A, B\rangle=\operatorname{tr}(A B)$, and for $G$ the orthogonal group $\mathbf{O}(k)$ acting on $M$ by conjugation. Then the space $\Sigma$ of diagonal matrices is a section. Moreover the symmetric group $S_{k}$ acts on $\Sigma$ by permuting the diagonal entries and the orbit spaces $M / G$ and $\Sigma / S_{k}$ are isomorphic as stratified sets. Quite generally it is good intuition to think of a section $\Sigma$ as representing a "canonical form" for elements of $M$; hence our title. Riemannian $G$-manifolds which admit sections are definitely the exception rather than the rule and they have many remarkable properties. The existence of sections for $M$ has important consequences for the invariant function theory, submanifold geometry, and $G$-invariant variational problems associated to $M$. While we do not know of earlier papers treating sections in generality, we have found several which treat important special cases. In particular when we showed G. Schwarz an early version of our results he pointed out to us a preprint of an important paper [Da2] by J. Dadok in which a detailed study is made (including a complete classification theorem) of orthogonal representations of compact connected Lie groups which admits sections (Dadok calls these polar representations). Later still we discovered two very interesting and much earlier papers by L. Conlon $[$ Co1, Co2 $]$ in which he considers Riemannian $G$-manifolds which admit flat, totally geodesic sections. This includes the case of polar representations, and Conlon came close to conjecturing Dadok's classification result. We will discuss in more detail later the results in these papers and how they relate to our own. We would like to thank Dadok for a number of helpful comments. It is clear not only that he

Received by the editors March 4, 1986.

1980 Mathematics Subject Classification (1985 Revision). Primary 57S15.

The first author was supported in part by NSF Grant No. MCS-8102696 and MSRI.

The second author was supported in part by NSF Grant No. DMS-8301928. 
had also discovered many of the facts reported here, but in some cases he probably knew them before we did.

One important application of sections is to invariant theory. We describe a wellknown class of examples: if a compact Lie group $G$ acts on its Lie algebra $\mathfrak{g}$ (Killing form as the inner product) by the adjoint action, then a maximal abelian subalgebra $\tau$ is a section, the Weyl group $W$ of $G$ acts on $\tau$, and $\mathfrak{g} / G \approx \tau / W$. Moreover the restriction map from the ring of $G$-invariant polynomials on $\mathfrak{g}$ to the ring of $W$-invariant polynomials on $\tau$ is an isomorphism; this is the Chevalley restriction theorem. These properties of the adjoint action can be generalized to arbitrary $G$-manifolds which admit sections. Namely if $\Sigma$ is a section of a Riemannian $G$ manifold $M$, then there exists a finite group $W$ acting on $\Sigma$ such that for each $\sigma$ in $\Sigma, \Sigma \cap G \sigma=W \sigma$ (so in particular $W \sigma \rightarrow G \sigma$ is a bijection $\Sigma / W \approx M / G$ ); and the $C^{\infty}$ version of the Chevalley restriction theorem holds. This reduces the theory of $G$-invariant functions on $M$ to the simpler invariant theory of $\tau$ under a finite group.

A second application is to the Riemannian geometry of submanifolds. The principal horizontal distribution $\nVdash$ is a distribution defined on the set $M^{0}$ of $G$-regular points by $\mathcal{H}(x)=\nu(G x)_{x}$. Then it is easily seen that $M$ admits sections if and only if $\not$ is integrable and $\exp _{x}\left(\nu(G x)_{x}\right)$ is a closed, properly embedded submanifold of $M$ (which is automatically totally geodesic). If $v \in \nu(G x)_{x}$ then $\tilde{v}(g x)=d g_{x}(v)$ defines a $G$-equivariant normal field on the principal orbit $G x$, and we say $\tilde{v}$ is $\pi$-parallel. Since $G x$ is a submanifold of $M$, there is an induced normal connection from the Riemannian connection of $M$, which defines another parallelism for $\nu(G x)$. In general these two parallelisms are different, and in fact they are the same if and only if $\mathcal{H}$ is integrable. In this case a principal orbit $N=G x$ as a submanifold of $M$ has the following properties:

(1) $\nu(N)$ is flat with trivial holonomy,

(2) $\exp _{y}\left(\nu(N)_{y}\right)$ is a totally geodesic submanifold of $M$ for all $y$ in $N$,

(3) the principal curvatures of $N$ along any parallel normal field are constant.

We note that the orbit foliation of $M$ is determined by a single principal orbit, and is the same as the parallel foliation of $N$ in $M$, i.e. $\left\{N_{v} \mid v \in \nu(N)_{x}\right\}$, where $N_{v}=\{y+\tilde{v}(y) \mid y \in M\}$. A submanifold $N$ of a space form $\mathbb{R}^{n}$ satisfying (i)-(iii) is called isoparametric $[\mathbf{T}]$. So it follows that if $G$ acting on $\mathbb{R}^{n}$ admits sections then the principal orbits are isoparametric. Conversely we show that if $N$ is isoparametric in $\mathbb{R}^{n}$ and is an orbit of a subgroup $G$ of $\mathbf{O}(n)$ then $N$ must be a principal $G$-orbit and the $G$-action on $\mathbb{P}^{n}$ admits sections. Then by Dadok's classification theorem of polar representation we conclude that every homogeneous isoparametric submanifold of $\mathbb{R}^{n}$ or $S^{n}$ is a principal orbit of the isotropy representation of some symmetric space $G_{1} / K$. There are infinitely many isoparametric submanifolds of Euclidean spaces of codimension two, which do not arise as an orbit of some polar representation [FKM, OT]. However there always exists a Weyl group for such submanifolds, and the parallel foliation gives an orbitlike foliation. Therefore the theory of isoparametric submanifolds can be generalized to arbitrary Riemannian manifolds (using (1)-(3) as definition), which can be thought of as a purely geometric analogue of the theory of Riemannian $G$-manifolds with sections.

The third application we have in mind is to the calculus of variations. We first recall the simple example of finding a harmonic function $u$ on $\mathbb{R}^{n}$. We must in 
principle solve a partial differential equation $\Delta u=0$ in $n$-independent variables. However if we know that $u$ is invariant under the group $\mathbf{O}(n)$ of rotations, then we can write $u(x)=f(\|x\|)$ and reduce the problem to the easily solved ordinary differential equation $\partial / \partial r\left(r^{n-1} \partial f / \partial r\right)=0$ on the half line $\mathbb{R}^{+}$. This is a classic example of a general and powerful method, called variously "reduction of variables" or the "cohomogeneity method", for attacking a broad class of problems in geometry and analysis (cf. [HHS, Hs1, Hs2, HL, PT]). In the general setting, we have as above a $G$-manifold $M^{n}$ and would like to study some class $\mathfrak{S}$ of $G$-invariant objects associated to $M$. Frequently one can set up a natural bijection between $\mathfrak{S}$ and some set $\tilde{\mathfrak{S}}$ of related objects attached to the orbit space $\tilde{M}$, so that if $M$ has cohomogeneity $k$ (i.e. $\operatorname{dim}(\tilde{M})=k$ ) we have effectively reduced a problem with $n$ independent variables to a generally easier problem with only $k$ independent variables. A serious difficulty in applying this method comes from the existence of the set $\tilde{M}_{s}$ of singular (i.e. lower dimensional) orbits. In general $\tilde{M}$ is not a smooth manifold but only a stratified set. The principal stratum $\tilde{M}-\tilde{M}_{s}$ (the set of principal orbits) is an open, dense, smooth $k$-dimensional manifold. $\tilde{M}_{s}$ is the (finite) union of the other orbit types of $M$, each of which is by itself a smooth manifold of dimension less than $k$, but in general $\tilde{M}$ has bad singularities along $\tilde{M}_{s}$, making it hard to study global analytical problems on $\tilde{M}$. The study of $\tilde{\mathfrak{S}}$ usually leads to solving some partial differential equation on $\tilde{M}-\tilde{M}_{s}$ together with complicated "boundary behavior" as we approach $\tilde{M}_{s}$. To circumvent the difficulties associated to the latter one can try to "resolve" the singularities along $\tilde{M}_{s}$, and an excellent way to do this is to choose (if one exists) a section $\Sigma$ for $M$ as above. Then the analysis of $\tilde{\mathfrak{S}}$ leads to solving a partial differential equation on the smooth $k$-manifold $\Sigma$ (rather than on the singular $k$-manifold $\tilde{M}$ ) and the complicated boundary behavior along $M_{s}$ is replaced by the generally more tractable problem of $W$-invariance. (For example in our example of harmonic functions on $M=\mathbb{R}^{n}$ with $G=\mathbf{O}(n)$, where $\tilde{M}=\mathbb{R}^{+}=[0, \infty)$ and $\tilde{M}_{s}=\{0\}$, we can take for $\Sigma$ any line $\{r e \mid r \in \mathbb{R}\}$ with $e$ in $S^{n-1}$ and $W=\mathbb{Z}_{2}$ (generated by $r e \rightarrow-r e$ ), so that instead of solving $\partial / \partial r\left(r^{n-1}(\partial f / \partial r)\right)=0$ on $\mathbb{R}^{+}$with certain boundary behavior at 0 , we solve it on $\mathbb{R}$ but accept only even solutions).

It is not hard to see that our definitions and theorems concerning Riemannian $G$-manifolds with sections generalize easily if we drop the assumption that the Lie group $G$ is compact and replace it with the weaker assumption that $G$ acts properly on $M$ (which is equivalent to the condition that there exists a $G$-invariant Riemannian metric on $M$ with $G$ being a closed subgroup of Iso $(M)$ ). Because many variational problems in geometry and physics are invariant under an infinite dimensional Lie group of "gauge transformations", another very interesting direction of generalization, about which little is yet known, is to develop an analogous canonical form theory for infinite dimension manifolds with infinite dimensional Lie group actions. In the case of the group of diffeomorphisms acting on the space of Riemannian metrics and the group $\mathcal{G}$ of gauge transformations acting on the space $A$ of connections of a principal bundle it is known that the actions are proper and that they admit local slices, so the possibility of sections existing, at least in special cases, seems quite reasonable. Moreover in the latter case doing a path integral over a section would clearly be easier than doing one over the moduli space $\mathcal{A} / \mathcal{G}$.

This paper is organized as follows: we set the terminology and review basic 
properties of $G$-manifolds and Riemannian $G$-manifolds in $\S 1$ and $\S 2$, and in $\S 3$ we develop some elementary properties of sections; we discuss the generalized Weyl group and the $C^{\infty}$ Chevalley restriction theorem for a Riemannian $G$-manifold which admits sections in $\S 4$; and in $\S 5$ we prove that if $M$ is a $G$-manifold and the principal isotropy subgroup $H$ is open in its normalizer $N(H)$ then the fixed point set $\Sigma$ of $H$ is a section with respect to any $G$-invariant metric on $M$, i.e. the section depends only on the pair $(G, H)$. Finally in $\S 6$ we discuss the submanifold geometry of the orbits of Riemannian $G$-manifolds $M$ which admit sections.

1. G-manifolds. In this section we establish our notation and review the basic theory of smooth transformation groups. Most details and proofs are omitted and may be found in $[\mathbf{B}, \mathbf{D}$, and $\mathbf{S 2}]$.

$G$ will denote a compact Lie group and $M$ a connected, smooth (i.e. $C^{\infty}$ ) $G$ manifold. For $x$ in $M$ we denote its orbit by $G x$ and its isotropy group by $G_{x}$. We denote the orbit space $M / G$ with the identification space topology by $\tilde{M}$ and $\Pi: M \rightarrow \tilde{M}$ is the orbit map. The conjugacy class of a closed subgroup $H$ of $G$ will be denoted by $(H)$ and is called a $G$-orbit type; the orbit $G x$ is said to be of type $(H)$ if $\left(G_{x}\right)=(H), M_{(H)} \subseteq M$ denotes the union of all orbits of type $(H)$, and $\tilde{M}_{(H)} \subseteq \tilde{M}$ its image in $\tilde{M}$ (the set of all orbits of type $(H)$ ). The fixed point set of $H$, i.e. the set of those $x$ in $M$ with $H \subseteq G_{x}$, will be denoted as usual by $M^{H}$, and $M_{H}$ will denote the set of $x$ in $M$ where $G_{x}$ is equal to $H$ (so $\left.M_{H}=\left(M_{(H)}\right)^{H}=M_{(H)} \cap M^{H}\right)$. From the fact that $G_{g x}=g G_{x} g^{-1}$ it follows that $g M_{H}=M_{g H g^{-1}}$. On the other hand if $N(H)$ denotes the normalizer of $H$ in $G$ then $g H^{-1}$ depends only on the coset $g N(H)$ of $g$ in $G / N(H)$. It follows that we have a well-defined map $p: M_{(H)} \rightarrow G / N(H)$ with $p^{-1}(g N(H))=M_{g H g^{-1}}$. In fact it is not hard to see that each $M_{(H)}$ is a smooth regularly embedded (but usually not closed) submanifold of $M$, and that $p$ is a smooth fiber bundle with fiber $M_{H}$ associated to the principal fibration $G \rightarrow G / N(H)$, with structure group $N(H) / H$.

In case $M$ has a single orbit type there is a canonical differentiable structure for $\tilde{M}$ making $\Pi: M \rightarrow \tilde{M}$ a submersion (and in fact a fiber bundle). But as just remarked, in general each $M_{(H)}$ is a smooth submanifold of $M$ and hence a smooth $G$-manifold in its own right, so each $\tilde{M}_{(H)}$ has a canonical differentiable structure. In fact these decompositions of $M$ and $\tilde{M}$ into submanifolds are "nice" (technically $M$ and $\tilde{M}$ are both stratified sets and $\Pi: M \rightarrow \tilde{M}$ is a stratified submersion). This fact has played an important role in the recent history of the subject. We refer to [D] for details.

Among all orbit types $(H)$ with $M_{(H)} \neq \varnothing$ there is a unique one $(U)$ such that $G / U$ has maximum dimension and (for that dimension) a maximum number of components. The orbit type $(U)$ is called the principal orbit type of $M$, any representative $U$ is called a principal isotropy group, and $M_{(U)}$ is called the principal stratum of $M$. To avoid having to name $(U)$ we will also write $M^{0}$ and $\tilde{M}^{0}$ for $M_{(U)}$ and $\tilde{M}_{(U)}$ respectively. The nonprincipal orbits of $M$ are called singular orbits and their union $M_{s}$ (the complement of $M^{0}$ ) is called the singular set of $M$. Thus $M_{s}$ and $\tilde{M}_{s}$ are closed and nowhere dense in $M$ and $\tilde{M}$ respectively. Points of $M^{0}$ are called regular points and points of $M_{s}$ singular points.

By choosing any Riemannian structure for $M$ and averaging it with respect to the Haar measure of $G$ we can always find an invariant Riemannian metric for $M$, 
i.e. one for which $G$ acts by isometries. Such a metric is also called compatible with the action of $G$, and $M$ with such a metric is called a Riemannian $G$-manifold. The differential of the action of $G_{x}$ defines a linear representation of $G_{x}$ on $T M_{x}$ called the isotropy representation at $x$. Since the tangent space $T(G x)_{x}$ to the orbit of $x$ is clearly an invariant linear subspace, we can find a complementary invariant subspace $\nu(G x)_{x}$ (e.g. the orthogonal complement to $T(G x)_{x}$ with respect to a compatible metric), and the restriction of the isotropy representation of $G_{x}$ to $\nu(G x)_{x}$ is called the slice representation at $x$. The image of a small ball in $\nu(G x)_{x}$ under the exponential map (with respect to a compatible metric) is a smooth $G_{x}$ invariant disk $\mathfrak{S}$ in $M$ called a slice at $x$. It meets all nearby orbits transversally and has the important property that for $y$ in $\mathfrak{S}$ the isotropy group $G_{y}$ is included in $G_{x}$. It follows easily that $x$ is a regular point if and only if the slice representation is trivial, or equivalently if and only if $\mathfrak{S}$ is pointwise fixed under $G_{x}$.

2. Riemannian submersions and Riemannian $G$-manifolds. If $\Pi: E \rightarrow$ $B$ is a submersion of smooth manifolds then $V=\operatorname{ker}(d \Pi)$ is a smooth subbundle of $T E$ called the tangent bundle along the fiber (or the vertical subbundle). In case $E$ and $B$ are Riemannian we define the horizontal subbundle $\not$ of $T E$ to be the orthogonal complement $V^{\perp}$ of the vertical bundle, and $\Pi$ is called a Riemannian submersion if $d \Pi$ maps $\nvdash_{x}$ isometrically onto $T B_{\Pi(x)}$ for all $x$ in $E$. The theory of Riemannian submersions, first systematically studied by O'Neill $[\mathbf{O}]$, plays an important role in the study of transformation groups. In this section we will discuss some basic geometric properties of Riemannian submersions and Riemannian $G$ manifolds.

A vector field $\xi$ on $E$ is called vertical (resp. horizontal) if $\xi(x)$ is in $V(x)$ (resp. $\mathcal{H}(x)$ ) for all $x$ in $E$, and $\xi$ is called projectable if there exists a vector field $\eta$ on $B$ such that $d \Pi(\xi)=\eta$. We call $\xi$ basic if it is both horizontal and projectable.

Note that if $F=\Pi^{-1}(y)$ is a fiber of $\Pi$ then $\forall \mid F$ is just the normal bundle $\nu(F)$ to $F$ in $E$. There is a canonical global parallelism in each such normal bundle $\nu(F)$ : a section $\tilde{v}$ of $\nu(F)$ is called $\Pi$-parallel if $d \Pi(\tilde{v}(x))$ is a fixed vector $v \in T B_{y}$ independent of $x$ in $F$. Clearly $\tilde{v} \rightarrow v$ is a bijective correspondence between $\Pi$ parallel fields and $T B_{y}$. There is another standard parallelism on $\nu(F)$ obtained from the Riemannian structure of $E$. Let $\hat{\nabla}$ denote the Levi-Civita connection of $E$, then the induced normal connection $\tilde{\nabla}$ on $\nu(F)$ is defined as follows:

$$
\tilde{\nabla}_{X} \xi=\text { the orthogonal projection on } \hat{\nabla}_{X} \xi \text { onto } \nu(F)
$$

where $X \in C^{\infty}(T F)$ and $\xi \in C^{\infty}(\nu(F))$. A normal vector field $\xi$ on $F$ is called parallel if $\tilde{\nabla} \xi=0$. It is important to note that in general the II-parallelism in $\nu(F)$ has no relation to the parallel translation defined by the Riemannian connection in $\nu(F)$. (The latter is in general not flat, while the former is always both flat and without holonomy.) Nevertheless we shall see that if $\forall$ is integrable then these two parallelisms do coincide. To prove this we need some basic results in the theory of Riemannian submersions.

2.1. TheOREM ( O'NeILl $[\mathbf{O}]$ ). Let $\Pi: E \rightarrow B$ be a Riemannian submersion, and $*$ its horizontal distribution.

(i) If $X$ is a vertical field and $Y$ is a basic field on $E$ then $[X, Y]$ is vertical.

(ii) If $\sigma$ is a geodesic in $E$ and $\sigma^{\prime}(0)$ is horizontal then $\sigma^{\prime}(t)$ is horizontal for all $t$ and $\Pi \circ \sigma$ is a geodesic in $B$. 
(iii) If $\mathcal{H}$ is integrable then the leaves are totally geodesic.

(iv) If $\forall$ is integrable and $S$ is a leaf of $\forall$ then $\Pi \mid S$ is a local isometry.

2.2. Definition. A Riemannian submersion $\Pi: E \rightarrow B$ is called integrable if the horizontal distribution $\forall$ is integrable.

2.3. ThEOREM. Let $\Pi: E \rightarrow B$ be a Riemannian submersion. Then $\Pi$ is integrable if and only if every $\Pi$-parallel vector field on $\Pi^{-1}(b)$ is a parallel normal field in the Riemannian sense (i.e. it is parallel with respect to the induced normal connection of $\left.\Pi^{-1}(b)\right)$.

PROOF. Let $e_{A}$ be a local orthonormal frame field on $E$ such that $e_{1}, \ldots, e_{n}$ are basic vector fields and $e_{n+1}, \ldots, e_{n+m}$ are vertical vector fields. We will use the index conventions $1 \leq i, j \leq n, n+1 \leq \alpha, \beta \leq n+m, 1 \leq A, B \leq n+m$, and we will write $\nabla_{i}$ for $\nabla_{e_{i}}, \ldots$, etc. Let $\omega_{A B}$ be the Levi-Civita connection on $M$, i.e. $\nabla e_{A}=\sum \omega_{A B} \otimes e_{B}$, and suppose $\Pi$ is integrable. Then by 2.1(iii) each leaf $S$ of the horizontal distribution $\forall$ is totally geodesic and $e_{i} \mid S$ is a local frame field on $S$. Thus the second fundamental form of $S$ is zero, i.e. $\omega_{i \alpha}\left(e_{j}\right)=0$ for all $i$ and $j$, or equivalently $\nabla_{j} e_{\alpha}$ is vertical. But $e_{\alpha} \mid F$ forms a tangent frame field for the fiber $F$ of $\Pi$, and $e_{i} \mid F$ is a normal vector field of $F$. Since $\left[e_{j}, e_{\alpha}\right]=\nabla_{j} e_{\alpha}-\nabla_{\alpha} e_{j}$ is vertical, we have $\nabla_{\alpha} e_{j}$ is vertical, i.e. $e_{j} \mid F$ is parallel in the normal connection of $\nu(F)$.

Conversely suppose $e_{i} \mid F$ is parallel for every fiber $F$ of $\Pi$, i.e. $\nabla_{\alpha} e_{j}$ is vertical. Since $\left[e_{i}, e_{\alpha}\right]$ is vertical, $\nabla_{j} e_{\alpha}$ is vertical, i.e. $\omega_{\alpha i}\left(e_{j}\right)=0$ for all $i$ and $j$. Now we note that

$$
\left[e_{i}, e_{j}\right]=\nabla_{i} e_{j}-\nabla_{j} e_{i}=\sum\left(\omega_{j A}\left(e_{i}\right)-\omega_{i A}\left(e_{j}\right)\right) e_{A} .
$$

Hence $\left[e_{i}, e_{j}\right]$ is horizontal, so $\not$ is integrable.

Henceforth $M$ will denote a connected, complete smooth Riemannian $G$-manifold. As noted in the preceding section for each orbit type $(K)$ the restricted orbit map $\Pi_{(K)}: M_{(K)} \rightarrow \tilde{M}_{(K)}$ is a submersion. We note that there is a canonical choice of Riemannian structure for $\tilde{M}_{(K)}$ making $\Pi_{(K)}$ a Riemannian submersion (so that $\Pi: M \rightarrow \tilde{M}$ is a stratified Riemannian submersion). To see this we can without loss of generality assume $M=M_{(K)}$. If $x \in M$ then we must of course define the inner product in $T \tilde{M}_{\pi(x)}$ by requiring that $d \Pi: \nu(G x)_{x} \rightarrow T \tilde{M}_{\pi(x)}$ is an isometry. Since $d g$ maps $\nu(G x)_{x}$ isometrically onto $\nu(G x)_{g x}$ this is well defined and is easily seen to give a smooth metric on $\tilde{M}$. Thus in particular we have a Riemannian submersion on the principal stratum $\Pi: M^{0} \rightarrow \tilde{M}^{0}$.

2.4. Definition. The principal horizontal distribution of a Riemannian $G$ manifold $M$ is the horizontal distribution of the Riemannian submersion on the principal stratum $\Pi: M^{0} \rightarrow \tilde{M}^{0}$.

If $x$ is a regular point of $M$ then the orbit $G x$ is a fiber of $\Pi$ and hence we have as above a well-defined global parallelism in $\nu(G x)$, the $\Pi$-parallelism. In this case the $\Pi$-parallelism has a simple group theoretic interpretation. Since $x$ is regular, the slice representation of $G_{x}$ on $\nu(G x)_{x}$ is trivial, which implies that $d g: \nu(G x)_{x} \rightarrow \nu(G x)_{g x}$ is well defined (i.e. does not depend on the choice of element in the coset $\left.g G_{x}\right)$. Thus any element $v_{x} \in \nu(G x)_{x}$ gives rise to a well-defined $G$ invariant section $\tilde{v}$ of $\nu(G x)$. Moreover since $\Pi \circ g=\Pi, d \Pi \circ d g=d \Pi$ and hence $\tilde{v}$ is $\Pi$-parallel, i.e. the П-parallelism is just given by group translation. (A word of 
caution: for a non principal stratum $M_{(H)}$ we again have a Riemannian submersion $\Pi_{(H)}: M_{(H)} \rightarrow M_{(H)}$ and hence a $\Pi_{(H)}$-parallelism on the normal bundle $\nu_{(H)}(G x)$ of an orbit $G x$ of type $(H)$. But note that $\nu_{(H)}(G x)$ denotes the normal bundle of $G x$ in $M_{(H)}$, a subbundle of $\nu(G x)$, its normal bundle in $M$.)

It will be convenient to introduce for each regular point $x$ the set $\tau(x)$, defined as the image of $\nu(G x)_{x}$ under the exponential map of $M$ and also $\tau^{0}(x)=\tau(x) \cap M^{0}$ for the set of regular points of $\tau(x)$.

2.5. Proposition. For each regular point $x$ of $M:$ (i) $g \tau(x)=\tau(g x)$ and $g \tau^{0}(x)=\tau^{0}(g x)$ for all $g \in G$,

(ii) for $v_{0} \in \nu(G x)_{x}$ the geodesic $\sigma(t)=\exp \left(t v_{0}\right)$ is orthogonal to each orbit it meets,

(iii) $T(x)$ meets every orbit of $M$.

ProOF. Statement (i) is obvious, and (ii) follows from 2.1(ii) and the fact that $\Pi: M^{0} \rightarrow \tilde{M}^{0}$ is a Riemannian submersion. Finally given any $y$ in $M$ choose $g \in G$ so that $g y$ minimizes the distance from $x$ to $G y$ and define $\sigma(t)=\exp \left(t v_{0}\right)$, a minimizing geodesic from $x=\sigma(0)$ to $g y=\sigma(1)$. Since $G$ acts isometrically, $\sigma$ is even a minimizing geodesic from $G x$ to $G y$, and hence $v_{0}=\sigma^{\prime}(0)$ and $\sigma^{\prime}(1)$ are orthogonal to $G x$ and $G y$ respectively. In particular $v_{0}$ is in $\nu(G x)_{x}$ so the arbitrary orbit $G y$ meets $\tau(x)=\exp \left(\nu(G x)_{x}\right)$ at $\exp \left(v_{0}\right)=g y$.

Our choice of the notation $\tau(x)$ is based on the fact that when $M$ is $G$ itself with the adjoint action, then $\tau(x)$ is just the maximal torus through the regular point $x$. Thus (iii) is a generalization of the fact that every element of a compact connected Lie group is conjugate to an element on a fixed maximal torus.

3. Sections and their elementary properties. Henceforth $M$ will denote a connected, complete, Riemannian $G$-manifold and we assume all the previous notational conventions. In particular we identify the Lie algebra $\mathfrak{g}$ of $G$ with the Killing fields on $M$ generating the action of $G$.

3.1. DEFinition. A connected, closed, regularly embedded smooth submanifold $\Sigma$ of $M$ is called a section for $M$ if it meets all orbits orthogonally.

The conditions on $\Sigma$ are, more precisely, that $G \Sigma=M$ and that for each $x$ in $\Sigma, T \Sigma_{x}$ is included in $\nu(G x)_{x}=T(G x)_{x}^{\perp}$. But since $T(G x)_{x}$ is just the set of $\xi(x)$ where $\xi \in \mathfrak{g}$, this second condition has the more explicit form

$(*)$ For each $x$ in $\Sigma$ and $\xi$ in $\mathfrak{g}, \xi(x)$ is orthogonal to $T \Sigma_{x}$.

It is trivial that if $\Sigma$ is a section for $M$ then so is $g \Sigma$ for each $g$ in $G$. Since $G \Sigma=M$, it follows that if one section $\Sigma$ exists then in fact there is a section through each point of $M$, and we shall say that $M$ admits sections.

If $\Sigma$ is a section for $M$ then the set $\Sigma^{0}=\Sigma \cap M^{0}$ of regular points of $\Sigma$ is an integral manifold of the principal horizontal distribution $\nLeftarrow$ of the $G$-action. It is known (see $\left[\mathbf{D}\right.$, Theorem 1.7]) that $\tilde{M}^{0}$ is always connected, so from 2.3 it follows that:

3.2. THEOREM. If $M$ admits sections, then

(i) the principal horizontal distribution $*$ is integrable;

(ii) if $\Sigma$ is a section for $M$ then each connected component of $\Sigma^{0}=\Sigma \cap M^{0}$ is a leaf of $\not{H}$; 
(iii) if $F$ is the leaf of $\forall$ through a regular point $x$ then $\Pi \mid F$ is a covering isometry onto $\tilde{M}^{0}$;

(iv) $\Sigma$ is totally geodesic;

(v) there is a unique section through each regular point $x$ of $M$, namely $\tau(x)=$ $\exp \left(\nu(G x)_{x}\right)$.

3.3. REMARK. It is natural to suspect that, conversely to 3.2(i), if $\not /$ is integrable then $M$ admits sections. To get a counterexample take $M=S^{1} \times S^{1}$ and let $G=S^{1} \times\{e\}$ acting by translation. Let $\xi$ denote the vector field on $M$ generating the action of $G$ and let $\eta$ denote an element of the Lie algebra of $S^{1} \times S^{1}$ generating a nonclosed one parameter group $\gamma$. If we choose the invariant Riemannian structure for $M$ making $\xi$ and $\eta$ orthonormal then a section for $M$ would have to be a coset of $\gamma$, which is impossible since $\gamma$ is not closed in $M$. This also gives a counterexample to the natural conjecture that if a compact $G$-manifold $M$ has codimension 1 principal orbits then any normal geodesic to the principal orbit is a section. It is also natural to believe that if $\forall$ is integrable, then the leaf of $\forall$ can be extended to be a complete immersed totally geodesic submanifold of $M$, which meets every orbit orthogonally. However we can only prove this in the real analytic case. But the following is true:

3.4. Proposition. Let $x_{0}$ be a regular point of $M$, and $\tau=\exp \left(\nu\left(G x_{0}\right)_{x_{0}}\right)$. Suppose $\forall$ is integrable and $T$ is a closed, properly embedded submanifold of $M$. Then $T$ is a section.

ProOF. By 2.5(iii) it suffices to show that $\tau$ is orthogonal to $G x$ for all $x$ in $\tau$. Let $F$ denote the leaf of $\forall$ through $x_{0}$. Then $F$ is open in $T$, so $\tau$ is orthogonal to $G y$ for all $y$ in $F$. Now suppose $x \in \tau-F$, since $\exp _{x}: T \tau_{x} \rightarrow \tau$ is regular almost everywhere, there is an open neighborhood $\mathcal{O}$ of the unit sphere of $T \tau_{x}$ such that for all $v$ in $O$ there is an $r>0$ such that $\sigma_{v}(r)=\exp (r v)$ is in $F$. Then by 2.5(ii) $\sigma_{v}^{\prime}(0)$ is normal to $G x$.

It is known that any connected, properly embedded, totally geodesic submanifold of a simply connected, complete symmetric space can be extended uniquely to one that is complete (cf. [KN, Chapter 9, Theorem 4.3]). So we have

3.5. COROLlaRY. Let $M=G / K$ be a simply connected complete symmetric space, and $H$ a subgroup of $G$. Then the action of $H$ on $M$ admits sections if and only if the principal horizontal distribution of this action is integrable. In particular if the principal $H$-orbit is of codimension one then the $H$-action on $M$ has a section.

It follows from 2.3 that

3.6. THEOREM. The following statements are equivalent for a Riemannian G-manifold $M$ :

(i) the principal horizontal distribution $*$ is integrable,

(ii) for all regular points $x$, every $G$-invariant (i.e. I-parallel) normal vector field on the principal orbit $G x$ is parallel in the induced normal connection for the normal bundle $\nu(G x)$ in $M$ (i.e. in the Riemannian geometry sense),

(iii) for each regular point $x$ of $M$, if $S$ is the normal slice at $x$ then for all $\xi$ in $\mathfrak{g}$ and $s$ in $S, \xi(s)$ is normal to $S$. 
Following J. Dadok we shall say that an orthogonal representation space $V$ of $G$ is polar if it admits sections. As a consequence of 3.5 and 3.6 we have

3.7. PROPOSITION. Let $V$ be an orthogonal representation of $G, x$ a regular point of $V$, and $\Sigma$ the linear subspace of $V$ orthogonal to the orbit $G x$ at $x$. Then the following are equivalent:

(i) $V$ is polar,

(ii) $\Sigma$ is a section for $V$,

(iii) for each $v$ in $\Sigma$ and $\xi$ in $\mathfrak{g}, \xi(v)$ is normal to $\Sigma$.

4. The generalized Weyl group of a section. In this section $M$ is a connected, complete Riemannian $G$-manifold which admits sections, $x$ is a regular point of $M, U=G_{x}$ a principal isotropy group and $\Sigma$ the section for $M$ through $x$. We recall that a small enough neighborhood 0 of $x$ in $\Sigma$ is a slice at $x$ and so intersects each orbit near $G x$ in a unique point. Also recall that $G_{x}$ acts trivially on the section $\Sigma$.

In general given a closed subset $S$ of $M$ we let $N(S)$ denote the closed subgroup $\{g \in G \mid g S=S\}$ of $G$, the largest subgroup of $G$ which induces an action on $S$, and we let $Z(S)$ denote the kernel of this induced action, i.e. $Z(S)=\{g \in G \mid g s=s$ for all $s$ in $S$ \} is the intersection of the isotropy groups $G_{s}, s \in S$. Thus $N(S) / Z(S)$ is a compact group acting effectively on $S$. In particular when $S$ is a section $\Sigma$ then we denote $N(\Sigma) / Z(\Sigma)$ by $W=W(\Sigma)$ and call it the generalized Weyl group of $\Sigma$, and we put $\tilde{\Sigma}=\Sigma / W$.

4.1. REMARK. If $M$ is $G$ itself with the adjoint action then, for $H$ a subgroup of $G, N(H)$ and $Z(H)$ are respectively the normalizer and centralizer of $H$. If for $H$ we take a maximal torus $T$ of $G$ (which we shall see later is in fact a section) then $Z(T)=T$ and $W(T)=N(T) / T$ is the usual Weyl group.

4.2. REMARK. As remarked above $U=G_{x} \subseteq Z(\Sigma)$, and conversely from the definition of $Z(\Sigma)$ it follows that $Z(\Sigma) \subseteq U$, so $Z(\Sigma)=U$ and $W(\Sigma)=N(\Sigma) / U$. Moreover if $g \Sigma \subset \Sigma$ then $G_{g x}=g G_{x} g^{-1}=G_{x}$. Hence $N(\Sigma) \subset N(U)$ and $W(\Sigma) \subset$ $N(U) / U$.

4.3. PROPOSITION. The generalized Weyl group $W(\Sigma)$ of a section $\Sigma$ is a finite group. Moreover if $\Sigma^{\prime}$ is a second section for $M$ then $W\left(\Sigma^{\prime}\right)$ is isomorphic to $W(\Sigma)$ by an isomorphism which is well determined up to inner automorphism.

ProOF. If $g \in N(\Sigma)$ is near the identity then $g x \in 0$. Since 0 meets each orbit near $x$ in a unique point, $g x=x$, i.e. $g \in G_{x}=U=Z(\Sigma)$, so $Z(\Sigma)$ is open in $N(\Sigma)$ and hence $N(\Sigma) / Z(\Sigma)$ is discrete and so finite. If $\Sigma^{\prime}$ is a second section then $\Sigma^{\prime}=\gamma \Sigma$ and so $g \rightarrow \gamma g \gamma^{-1}$ clearly induces an isomorphism of $W(\Sigma)$ onto $W\left(\Sigma^{\prime}\right)$.

In [Co1] Conlon defines a $G$-transversal domain for a $G$-manifold $M$ to be a flat, closed, connected, totally geodesic submanifold of $M$ meeting every $G$-orbit and orthogonal to $G$-orbits at every point of intersection. As we have seen, meeting all $G$-orbits orthogonally (i.e. being a section) automatically implies totally geodesic, and so we can paraphrase Conlon's definition as saying a $G$-transversal domain is a flat section. Now in general being flat is a strong extra restriction on a section, but there is one case when it is clearly no restriction at all; namely when $M$ itself is flat. In particular for an orthogonal representation a $G$-transversal domain and a section are the same thing and so the representations with a $G$-transversal domain, 
studied by Conlon in $[$ Co2 $]$ are exactly the polar representations studied by Dadok [Da2].

As Conlon remarks, the earliest representations singled out as having $G$-transversal domains were noted by Bott and Samelson in [BS] and by R. Hermann in [H1, H2]. In particular it follows from [BS] (and is easily seen directly) that isotropy representations of symmetric spaces (called $s$-representations by Conlon) are polar. More precisely, if $U / G$ is a compact symmetric space and the Lie algebra $\mathfrak{u}$ of $U$ has the Cartan splitting $\mathfrak{u}=\mathfrak{g} \oplus \mathfrak{m}$, then a maximal abelian subalgebra $\mathfrak{a}$ of $\mathfrak{m}$ is a section for the adjoint representation of $G$ on $\mathfrak{m}$ (which of course is the isotropy representation of $U / G$ ) and the generalized Weyl group is the usual Weyl group associated to a symmetric space. Conlon also showed that polar representations shared many of the remarkable special properties of $s$-representations, while at the same time providing two explicit examples of polar representations which were not $s$-representations. The situation was finally greatly clarified in Dadok's recent paper $[\mathbf{D a 2}]$ in which polar representations of compact connected Lie groups are completely classified, and then using the classification the following theorem is proved, which makes explicit the intimate connection between $s$-representations and polar representations:

4.4. THEOREM (J. DADOK $[\mathbf{D a 2}])$. Let $\rho: H \rightarrow \mathbf{O}(n)$ be a polar representation of a compact connected Lie group. Then there exist an $n$-dimensional symmetric space $M=G / K$ and a linear isometry $A: \mathbb{R}^{n} \rightarrow T M_{e K}$ mapping $H$ orbits onto $K$-orbits.

4.5. EXAMPLES. $G$-manifolds which admit sections arise naturally in geometry. For example both the $K$-action on the symmetric space $M=G / K$ and the isotropy representation of $K$ on $T M_{e K}$ admit sections, and the generalized Weyl groups for these two actions are the same and equal to the standard Weyl group associated to $M$. However in general a generalized Weyl group is not a Coxeter group. In fact one can construct examples with arbitrary finite group as the generalized Weyl group. Given any compact group $G$, a closed subgroup $H$ of $G$, a finite subgroup $W$ of $N(H) / H$, and a smooth manifold $\Sigma$ such that $W$ acts faithfully on $\Sigma$, we let $\Pi: N(H) \rightarrow N(H) / H$ be the natural projection map, and $K=\Pi^{-1}(W)$, so $K$ acts naturally on $\Sigma$. Let $M=G \times_{K} \Sigma=\{(g, \sigma) \mid g \in G, \sigma \in \Sigma\} / \sim$ where the equivalence relation $\sim$ is defined by $(g, \sigma) \sim\left(g k^{-1}, k \sigma\right)$, and define the $G$-action on $M$ by $\gamma(g, \sigma)=(\gamma g, \sigma)$. Now suppose $d s^{2}$ is a metric on $M$ such that $d s^{2} \mid \Sigma$ and $d s^{2} \mid \nu(\Sigma)$ are $K$-invariant. Then $G$ acts on $M$ isometrically with $\Sigma=e \times \Sigma$ as a section, $(H)$ as the principal orbit type, and $W$ as the generalized Weyl group. Note that any finite group $W$ can be embedded as a subgroup of $\mathbf{S O}(n)$ for some $n$. Then the above construction gives a $G$-manifold admitting sections and having $W$ as the generalized Weyl group for $G=\mathbf{S O}(n), H=\{$ id $\}$, and $\Sigma=S^{n-1}$. Therefore it seems unlikely that there will be a good structure theory for general isometric actions admitting sections. However for special classes of Riemannian manifolds it is an interesting problem to classify all isometric actions which admit sections. Dadok's Theorem 4.4 gives a classification for the isometric actions of $\mathbb{P}^{n}$ and $S^{n}$ which admit sections. For arbitrary symmetric spaces, the classification is not yet known and finding one would be very interesting.

[Added in revision]. We learned recently that Szenthe also studied sections, which he called orthogonally transversal submanifolds, in $[\mathbf{S z 2}]$. Some of his results are 
equivalent to ours. In addition he obtained a structure theory for the generalized Weyl group (Theorem 4 of [Sz2]), but this seems to be incorrect, since the above construction implies that any finite group can be a generalized Weyl group.

4.6. THEOREM. If $M$ admits sections and $p \in M$ then the slice representation at $p$ is polar. In fact if $\Sigma$ is a section for $M$ through $p$ then $T \Sigma_{p}$ is a section and $W(\Sigma)_{p}=\{\varphi \in W(\Sigma) \mid \varphi(p)=p\}$ the generalized Weyl group for the slice representation at $p$.

PROOF. Let $V=\nu(G p)_{p}$ be the space of the slice representation, so that by definition of a section $T \Sigma_{p}$ is a linear subspace of $V$. Recall that for $v$ in $V$ its isotropy group $\left(G_{p}\right)_{v}$ in $G_{p}$ is the same as its full isotropy group $G_{v}$ in $G$, so that $G_{p} v$ is diffeomorphic to $G_{p} / G_{v}$ and in particular they have the same dimension. From this follows the well-known fact that a $G_{p}$ orbit in $V$ has the same codimension in $V$ as the corresponding $G$ orbit has in $M$, so in particular $V$ has the same cohomogeneity as a $G_{p}$-space as $M$ has as a $G$-space, namely $\operatorname{dim}(\Sigma)$. Thus $T \Sigma_{p}$ has dimension complementary to that of the principal $G_{p}$ orbits of $V$ and by the preceding proposition it will be enough to show that at each $v$ in $T \Sigma_{p}, T \Sigma_{p}$ is orthogonal to $G_{p} v$ (with respect of course to the flat metric in $V$ ). Let $v(t)=$ $\exp (t v)$. Since $\Sigma$ is a section for $M, T \Sigma_{v(t)}$ is orthogonal to $T(G v(t))_{v(t)}$ and a fortiori $T \Sigma_{v(t)}$ is orthogonal to $T\left(G_{p} v(t)\right)_{v(t)}$. But under the exponential map $\exp : T M_{p} \rightarrow M$ we can identify $T \Sigma_{v(t)}$ with $T \Sigma_{p}$ for all $t$ (because $\Sigma$ is totally geodesic) and also $T\left(G_{p} v(t)\right)_{v(t)}=T\left(G_{p} v\right)_{v}$ (since tangent spaces to orbits of linear representations are clearly constant along rays). In other words $T \Sigma_{p}$ and $T\left(G_{p} v\right)_{v}$ are orthogonal subspaces of $T M_{p}$ at the point $t v$ for all $t$ with respect to the (nonflat) metric on $T M_{p}$ pulled back from $M$ under $\exp : T M_{p} \rightarrow M$. But as $t \rightarrow 0$ this pulled back metric converges uniformly to the flat metric on $T M_{p}$, so $T \Sigma_{p}$ and $T\left(G_{p} v\right)_{v}$ are also orthogonal in the flat metric. It remains to prove that $W\left(T \Sigma_{p}\right)=W(\Sigma)_{p}$. To see this we note that $N\left(T \Sigma_{p}\right)=N(\Sigma) \cap G_{p}$ and $Z\left(T \Sigma_{p}\right)=Z(\Sigma) \cap G_{p}=Z(\Sigma)$, so $W\left(T \Sigma_{p}\right) \subset W(\Sigma)_{p}$. Conversely if $g Z(\Sigma) \in W_{p}$, then $g p=p$, which implies that $W(\Sigma)_{p} \subset W\left(T \Sigma_{p}\right)$.

4.7. COROLlaRY. If $G$ is a compact connected Lie group and $M$ is a $G$ manifold which admits sections and has a fixed point $p$, then the generalized Weyl group for $M$ is a Weyl group.

4.8. COROLlary. If $M$ admits sections then for any $p$ in $M$ the isotropy group $G_{p}$ acts transitively on the set of sections of $M$ which contain $p$.

PROOF. Let $\Sigma_{1}$ and $\Sigma_{2}$ be sections through $p$ and let $x$ be a regular point of $\Sigma_{1}$ near $p$. We may regard $\Sigma_{2}$ as a section for the slice representation at $p$, so that it meets $G_{p} x$, i.e. there exists $g$ in $G_{p}$ such that $g x \in \Sigma_{2}$. Since $g \Sigma_{1}$ and $\Sigma_{2}$ are both sections of $M$ containing the regular point $x$ they are equal by 3.2(5).

4.9. COROLlaRY. If $\sigma \in \Sigma$ then its orbit $W(\Sigma) \sigma$ under the generalized Weyl group is equal to the intersection of its $G$-orbit $G \sigma$ with $\Sigma$.

Proof. Since $W(\Sigma) \sigma=N(\Sigma) \sigma \subseteq G \sigma$, it is clear that $W(\Sigma) \sigma \subseteq G \sigma \cap \Sigma$. Conversely suppose $\sigma^{\prime} \in G \sigma \cap \Sigma$; say $\sigma^{\prime}=g \sigma$. Then $g \Sigma$ is a section at $\sigma^{\prime}$ so by 4.4 there is a $\gamma$ in $G_{\sigma^{\prime}}$ such that $\gamma g \Sigma=\Sigma$. Thus $(\gamma g) \in N(\Sigma)$ so $\sigma^{\prime}=\gamma \sigma^{\prime}=\gamma g \sigma$ is in $N(\Sigma) \sigma=W(\Sigma) \sigma$. 
4.10. Corollary. The map $j: \tilde{M} \rightarrow \tilde{\Sigma}$ defined by $G x \rightarrow G x \cap \Sigma$ is a welldefined homeomorphism. In fact with respect to the stratifications of $\tilde{M}$ and $\tilde{\Sigma}$ by orbit types $j i$ an isomorphism of stratified sets. Its inverse $j^{-1}: \tilde{\Sigma} \rightarrow \tilde{M}$ is the $\operatorname{map} W \sigma \rightarrow G \sigma$.

ProOF. It is immediate from the definition of a section that $G x \cap \Sigma$ is nonempty, and then by Corollary 4.5 it is an orbit of $W$, so $j$ is well defined and, again by the lemma, so is its inverse $W \sigma \rightarrow G \sigma$. It is clear that $j^{-1}$ is continuous in the identification space topology and, since $\tilde{M}$ and $\tilde{\Sigma}$ are compact, it follows from Theorem 4.3 that $j$ is a homeomorphism. Finally it follows from 4.3 that two orbits of $M$ have the same $G$ type if and only if their $j$ images have the same $W$ type.

$G$ acts linearly on the Banach space $C^{0}(M)$ of continuous real values functions on $M\left((g \cdot f)(x)=f\left(g^{-1} x\right)\right)$ and we let $C^{0}(M)^{G}$ denote the closed linear subspace of $G$ invariant functions, i.e. those constant on $G$-orbits. Clearly the map $\Pi^{*}: C^{0}(\tilde{M}) \rightarrow$ $C^{0}(M), f \rightarrow f \circ \Pi$, maps $C^{0}(\tilde{M})$ isomorphically onto $C^{0}(M)^{G}$. Similarly we have an isomorphism $\pi^{*}: C^{0}(\tilde{\Sigma}) \rightarrow C^{0}(\Sigma)^{W}$. Then it follows immediately from 4.4 that

4.11. PROPOSITION. The restriction map $f \rightarrow f \mid \Sigma$ is an isomorphism $\rho$ : $C^{0}(M)^{G} \rightarrow C^{0}(\Sigma)^{W}$ of Banach algebras.

4.12. Theorem (Polar Representation Restriction Theorem). Let $V$ be a polar representation of $G, \Sigma$ a section of $V, W=W(\Sigma)$ the Weyl group of $\Sigma$, and let $\mathbb{R}[V]^{G}$ and $\mathbb{R}[\Sigma]^{W}$ denote respectively the rings of $G$-invariant polynomials on $V$ and $W$-invariant polynomials on $\Sigma$. Then the restriction map $f \rightarrow f \mid \Sigma$ is an isomorphism $\rho: \mathbb{R}[V]^{G} \rightarrow \mathbb{R}[\Sigma]^{W}$.

PROOF. The case that $V$ is the adjoint representation of $G$ on its Lie algebra (so $\Sigma$ is the Lie algebra of a maximal torus) is the classical Chevalley restriction theorem. The Chevalley theorem was generalized in $[\mathbf{T}]$ to the case of an arbitrary isoparametric foliation of $\mathbb{R}^{n}$, and as we shall see in $\S 6$ the orbit foliation of a polar representation is a special case (the homogeneous case) of an isoparametric foliations.

4.13. Corollary. If $F \in C^{0}(V)^{G}$ and $f=F \mid \Sigma$ is in $C^{\infty}(\Sigma)^{W}$ then $F$ is in $C^{\infty}(V)^{G}$.

PROOF. According to a theorem of G. Schwarz $[\mathbf{S 1}]$ there is a $C^{\infty}$ function $\varphi$ on $\mathbb{R}^{k}$ such that $f=\varphi\left(u_{1}, \ldots, u_{k}\right)$, where $u_{1}, \ldots, u_{k}$ are generators for $\mathbb{R}[\Sigma]^{W}$. By $4.7 u_{i}=U_{i} \mid \Sigma$, where $U_{i} \in \mathbb{P}[V]^{G}$, and hence $F=\varphi\left(u_{1}, \ldots, u_{k}\right)$ is $C^{\infty}$.

4.14. LeMma. Let $M$ be a Riemannian $G$-manifold and let $\Sigma$ be a section of $M$ containing a point $x$. If $S$ is the slice $\exp \left(\nu_{\varepsilon}(G x)_{x}\right)$ at $x$ and $\varphi$ is a $G_{x}$-invariant continuous function on $S$ such that $\varphi \mid(S \cap \Sigma)$ is $C^{\infty}$ then $\varphi \mid S$ is $C^{\infty}$.

PROOF. The exponential map is a $G_{x}$ equivariant diffeomorphism of the $\varepsilon$-ball in $V=\nu(G x)_{x}$ (the space of the slice representation at $x$ ) onto $S$, and since $\Sigma$ is totally geodesic it maps the $\varepsilon$-ball in $T \Sigma_{x}$ diffeomorphically onto $S \cap \Sigma$. The result is then immediate from 4.12 and 4.13 . 
4.15. THEOREM ( $C^{\infty}$ RESTRICTION THEOREM FOR $G$-MANifoldS With SECTIONS). Let $\Sigma$ be a section for the Riemannian $G$-manifold $M$ and let $W=$ $W(\Sigma)$ be its generalized Weyl group. Then the restriction isomorphism $C^{0}(M)^{G} \rightarrow$ $C^{0}(\Sigma)^{W}$ induces an isomorphism $C^{\infty}(M)^{G} \rightarrow C^{\infty}(\Sigma)^{W}$.

Proof. Let $F \in C^{0}(M)^{G}$ and suppose $f=F \mid \Sigma$ is $C^{\infty}$. We must show that $F$ is $C^{\infty}$ at an arbitrary $x$ in $M$. Let $S$ be a slice at $x$ as in 4.14. Since $S \times G x$ is diffeomorphic to a neighborhood of $x$ of $M$ (see Lemma 2.2 of $[\mathbf{P}]$ ), it will suffice to show that $\varphi=F \mid S$ is smooth, and so by 4.14 it will be enough to see that $\varphi \mid(S \cap \Sigma)$ is smooth. But since $\varphi|(S \cap \Sigma)=f| \Sigma$, this is clear.

This theorem was also proved by Dadok [Da1] for isotropy representations of symmetric spaces.

5. G-manifolds with canonical sections. Let $H$ be a closed subgroup of a compact Lie group $G$. If $M$ is any $G$-manifold then the stationary set $M^{H}$ of $H$ is a closed properly imbedded submanifold of $M$ which is totally geodesic with respect to any $H$-invariant metric. In case $M$ has $(H)$ as its principal orbit type then, as we shall see below, $\operatorname{dim}\left(M^{H}\right) \geq \operatorname{cohomogeneity}(M)$, and if equality holds then $M^{H}$ is a section for $M$ with respect to any $G$-invariant metric for $M$. Moreover the necessary and sufficient condition for this to hold is a condition solely on $H$ as a subgroup of $G$, namely that it be open in its normalizer $N(H)$, so that $W_{H}=N(H) / H$ (which turns out to be the generalized Weyl group of the section $M^{H}$ ) is finite. We now prove these facts.

Let $\mathfrak{h}$ be the subalgebra of $\mathfrak{g}$ corresponding to $H$ and consider $\mathfrak{g} / \mathfrak{h}$ as a linear $H$-space under the adjoint representation. As is well known and trivial $\mathfrak{g} / \mathfrak{h}$ is the isotropy representation of $H$ at the identity coset of $G / H$ so that more generally if $M$ is a $G$-space and $x \in M$ with $G_{x}=H$ then we can identify $\mathfrak{g} / \mathfrak{h}$ with the subrepresentation $T(G x)_{x}$ of the isotropy representation of $H$ on $T(M)_{x}$. Let $1_{H}$ denote the trivial one-dimensional representation of $H$ so that for an arbitrary linear $H$-space $V, \#\left(1_{H}, V\right)$, the multiplicity of $1_{H}$ in $V$, is equal to $\operatorname{dim}\left(V^{H}\right)$. Now $T\left(M_{x}\right)^{H}=\left(T M^{H}\right)_{x}$, and hence since $T M_{x}$ is the direct sum of $T(G x)_{x} \approx \mathfrak{g} / \mathfrak{h}$ and the slice representation $\nu(G x)_{x}$ :

$$
\operatorname{dim}\left(T M^{H}\right)_{x}=\#\left(1_{H}, \mathfrak{g} / \mathfrak{h}\right)+\#\left(1_{H}, \nu(G x)_{x}\right) .
$$

Now suppose that $x$ is a regular point of $M$ (i.e. $H$ is a principal isotropy group of $M)$, so that the slice representation of $H$ on $\nu(G x)_{x}$ is trivial. Then $\#\left(1_{H}, \nu(G x)_{x}\right)$ $=\operatorname{dim}\left(\nu(G x)_{x}\right)=$ cohomogeneity $(M)$. Thus

5.1. LEMMA. If $H$ is a principal isotropy group of a smooth $G$-manifold $M$ and $x$ is a regular point of $M^{H}$ then

$$
\operatorname{dim}\left(T M^{H}\right)_{x}=\#\left(1_{H}, \mathfrak{g} / \mathfrak{h}\right)+\operatorname{cohomogeneity}(M)
$$

5.2. LEMMA. Let $Z$ and $N$ (with Lie algebras $Z$ and $\mathcal{N}$ ) denote respectively the centralizer and normalizer of $H$ in $G$, so that $C=Z \cap H$ (with Lie algebra $\mathcal{C}=Z \cap \mathfrak{h})$ is the center of $H$. If $\mathfrak{a}$ is the orthogonal complement of $\mathfrak{h}$ in $\mathcal{N}$ with respect to an ad invariant metric, then

(1) $\mathfrak{a}$ is an ideal of $\mathcal{N}$.

(2) $\mathcal{N}=\mathfrak{h} \oplus \mathfrak{a}$.

(3) $Z=C \oplus$ a.

(4) $\mathcal{N} / \mathfrak{h}$ is the fixed point set of the adjoint representation of $H$ on $\mathfrak{g} / \mathfrak{h}$. 
Thus the following are equivalent:

(a) $\mathfrak{a}=0$.

(b) $\mathcal{N}=\mathfrak{h}$.

(c) $\boldsymbol{Z} \subseteq \mathfrak{h}$.

(d) The adjoint representation of $H$ on $\mathfrak{g} / \mathfrak{h}$ does not contain the trivial representation.

PROOF. With respect to the adjoint representation $\rho$ of $N$ on $\mathcal{N}$ the invariant linear subspaces are just the ideals of $\mathcal{N}$. Now $\rho$ is orthogonal with respect to an ad invariant metric and both $\mathcal{C}$ and $\mathfrak{h}$ are ideals of $\mathcal{N}$, so we have orthogonal decompositions

$$
\mathfrak{h}=\mathfrak{b} \oplus \mathfrak{C}, \quad \mathcal{N}=\mathfrak{a} \oplus \mathfrak{h}=\mathfrak{a} \oplus \mathfrak{b} \oplus \mathfrak{C}
$$

where $\mathfrak{a}$ and $\mathfrak{b}$ are ideals of $\mathcal{N}$. Since $\mathfrak{a}$ and $\mathfrak{h}$ are both ideals of $\mathcal{N}$ they must commute, i.e. $\mathfrak{a} \subseteq Z$. We have the further orthogonal decomposition:

$$
Z=\mathfrak{a} \oplus \mathfrak{b}^{\prime} \oplus \mathcal{C} \subset \mathcal{N}
$$

and it follows that $\mathfrak{b}^{\prime} \subseteq \mathfrak{b} \subseteq \mathfrak{h}$, so $\mathfrak{b}^{\prime} \subseteq Z \cap \mathfrak{h}=\mathcal{C}$, and hence $\mathfrak{b}^{\prime}=0$, giving $Z=\mathfrak{a} \oplus \boldsymbol{C}$.

The canonical projection $\mathfrak{g} \rightarrow \mathfrak{g} / \mathfrak{h}$ induces by restriction an equivalence of the adjoint representation of $H$ on $\mathfrak{m}$ (the orthocomplement of $\mathfrak{h}$ in $\mathfrak{g}$ ) with the adjoint representation of $H$ on $\mathfrak{g} / \mathfrak{h}$. Since the fixed point set of $\operatorname{ad}(H)$ on $\mathfrak{g}$ is clearly $\boldsymbol{Z}=\mathfrak{a} \oplus \mathcal{C}, \mathfrak{a}$ is exactly the fixed point of $\operatorname{ad}(H)$ on $\mathfrak{m}$, so $\mathfrak{a} \oplus \mathfrak{h} / \mathfrak{h}=\mathcal{N} / \mathfrak{h}$ is the fixed point set of $\operatorname{ad}(H)$ on $\mathfrak{g} / \mathfrak{h}$.

5.3. THEOREM. Let $H$ be a closed subgroup of a compact Lie group $G$ and let $N$ and $Z$ (with Lie algebras $\mathcal{N}$ and $Z$ ) denote respectively the normalizer and centralizer of $H$ in $G$. Then the following are equivalent:

(i) $\mathrm{eH}$ is an isolated stationary point of the action of $H$ on $G / H$.

(ii) The adjoint representation of $H$ on $\mathfrak{g} / \mathfrak{h}$ does not contain the trivial representation.

(iii) $\mathcal{N}=\mathfrak{h}$. That is, $H$ is open in its normalizer.

(iv) $W_{H}=N / H$ is finite.

(v) $Z \subseteq \mathfrak{h}$. That is, the identity component of $Z$ is included in $H$.

(vi) Let $M=G / H$, then $\operatorname{dim}\left(M^{H}\right)=0$ and $M^{H}=W_{H}$.

(vii) Any smooth $G$-manifold with principal isotropy type $(H)$ admits sections, and for any regular point $x$ of $M, M^{G_{x}}$ is the section for $M$ through $x$.

Moreover if these conditions are true then for any $M$ as in (vii) the generalized Weyl group of $M^{H}$ is $W_{H}$.

Proof. Considering $G / H$ as an $H$-space the isotropy group at the coset $g H$ is $g H^{-1} \cap H$, so that $g H$ is a stationary point precisely when $g H^{-1}=H$, that is when $g \in N(H)$, so that the set of stationary points is exactly $W_{H}$. Then the equivalence of (i), (ii), and (iv) follows from Lemma 5.1. The equivalence of (ii), (iii), (iv), and (v) follows from Lemma 5.2.

Since the dimension of a section is equal to the cohomogeneity of $M$ it is clear that (vii) implies (ii) and to complete the proof we will show that (ii) implies (vii), and at the same time verify the final conclusion of the theorem. So let $M$ be a Riemannian $G$-manifold with principal isotropy type $(H)$, and with $\#\left(1_{H}, \mathfrak{g} / \mathfrak{h}\right)=0$, and let $x$ be a point of $M$ with $G_{x}=H$. Since $\operatorname{dim}\left(\nu(G x)_{x}\right)$ is the cohomogeneity of $M$, it follows 
from 5.1 that in fact $\nu(G x)_{x}=T\left(M^{H}\right)_{x}$. Since this is true at every regular point of $M^{H}$, the set of regular points of $M^{H}$ is an integral manifold for the principal horizontal distribution $\nLeftarrow$ of $M$. By homogeneity it follows that $\forall$ is integrable. Since $M^{H}$ is a closed, properly imbedded, totally geodesic submanifold of $M$ it now follows from 3.4 that $M^{H}$ is a section for $M$. Finally since $g M^{H}=M^{g H g^{-1}}$, it follows that $N\left(M^{H}\right)=N(H)$ so $W\left(M^{H}\right)=N(H) / H=W_{H}$.

5.4. DEFinition. A smooth $G$-manifold $M$ will be said to have canonical sections if a principal isotropy group $H$ satisfies the equivalent conditions of 5.3.

The important point to notice is that having canonical sections is a condition only on the type of the principal orbits. In particular $M$ is not even assumed to be Riemannian; if it has canonical sections then no matter what $G$-invariant metric we choose for $M$, not only will it have sections, but in fact the section containing a regular point $x$ of $M$ will necessarily be $M^{G_{x}}$. Therefore it is an interesting problem to classify all the pairs $(G, H)$ such that $H$ is open in its normalizer.

5.5. Corollary. If a principal isotropy group $H$ of a smooth $G$-manifold $M$ includes a maximal abelian subgroup $A$ of $G$ (in particular if $H$ has maximal rank) then $M$ has canonical sections.

Proof. By assumption $A$ is equal to its centralizer in $G$. But then since $A \subseteq H$, the centralizer $Z$ of $H$ is included in $A$, so $Z \subseteq H$ and (v) of the theorem is satisfied.

In [Sz1] J. Szenthe shows directly that if $H$ has maximal rank then (vii) of the theorem is satisfied. It should be noted however that for a $G$-manifold with principal orbits of type $H$ to have canonical sections it is not necessary that $H$ have maximal rank in $G ; G=\mathbf{S O}(2 n)$ and $H=\mathbf{S O}(2 n-1)$ is a simple counterexample. Also $G$ can have maximal abelian subgroups which are not tori (for example $\mathbf{S O}(2 n)$ has a maximal abelian subgroup $\left(\mathbf{Z}_{2}\right)^{n}$ ).

5.6. EXAMPLE. If for $M$ we take $G$ with the adjoint action $(g(\gamma)=\operatorname{ad}(g) \gamma=$ $\left.g \gamma g^{-1}\right)$ then the principal orbit type is the conjugacy class $(T)$ of the maximal tori of $G$. The stationary set $M^{T}$ of $T$ is just $T$ itself. Of course $T$ is open in its normalizer $N(T)$ and $W_{T}=N(T) / T$ is the Weyl group of $G$. Thus the adjoint action of any compact Lie group on itself has canonical sections which are the maximal tori of $G$ and the generalized Weyl group is just the usual Weyl group associated to $G$. The identity $e$ is a stationary point of $M$ and the isotropy representation of $G$ on $T M_{e}=$ $\mathfrak{g}$ is the adjoint representation $\operatorname{Ad}$. Now $\operatorname{ad}(g) \exp (X)=\exp (\operatorname{Ad}(g) X)$, i.e. exp is an equivariant diffeomorphism of a neighborhood of 0 in $\mathfrak{g}$ with a neighborhood of $e$ in $M$, so $X$ and $\exp (X)$ have the same isotropy group, and in particular $\mathfrak{g}$ also has principal isotropy type $(T)$, and hence is polar with canonical sections the maximal abelian subalgebras $\mathfrak{a}$ of $\mathfrak{g}$, and generalized Weyl group the Weyl group of $G$. It also follows easily from Corollary 5.5 that the above examples admit sections because $T$ has maximal rank in $G$.

6. The submanifold geometry of orbits when sections exist. If $M$ is a complete Riemannian $G$-manifold which admits sections, then the orbit foliation of $M$ has remarkable Riemannian geometric properties. In this section we will study some geometric aspects of these orbits as submanifolds of $M$, and we also will discuss to what extent the Riemannian geometry of one principal orbit can determine the whole orbit foliation. 
To set notations we review briefly some elementary submanifold theory. Let $M$ be a Riemannian manifold, and $\hat{\nabla}$ its Levi-Civita connection. Suppose $N$ is a submanifold of $M$. Then $N$ has an induced metric and the Levi-Civita connection $\nabla$ on $N$ is related to $\hat{\nabla}$ as follows:

$$
\nabla_{X} Y=\text { the orthogonal projection of } \hat{\nabla}_{X} Y \text { onto } T N .
$$

The shape operator $A_{v}$ of $N$ in the normal direction $v$ is defined to be the selfadjoint operator

$$
A_{v}(X)=-\left(\text { the orthogonal projection of } \hat{\nabla}_{X} v \text { onto } T N\right)
$$

and the eigenvalues of $A_{v}$ are called the principal curvatures of $N$ in the direction of $v$. The normal connection of $\nu(N)$ is defined as in $\S 2$ to be

$$
\tilde{\nabla}_{X} \xi=\text { the orthogonal projection of } \nabla_{X} \xi \text { onto } \nu(N) .
$$

A normal field $\xi$ is parallel if $\tilde{\nabla} \xi=0$, and $\nu(N)$ is flat if the curvature of the normal connection $\tilde{\nabla}$ is zero, which is equivalent to the condition that locally there exists a parallel orthonormal frame field for $\nu(N)$.

6.1. THEOREM. Suppose $M$ is a complete Riemannian $G$-manifold that admits sections, and $N$ is a principal orbit of $M$. Then

(0) $\exp \left(\nu(N)_{x}\right)$ is a properly embedded totally geodesic submanifold of $M$ for all $x \in N$.

(1) $\nu(N)$ is flat and has trivial holonomy; in fact if $v_{1}, \ldots, v_{k}$ is a basis for $\nu(N)_{x}$ then the $G$-invariant normal fields $\hat{v}_{i}(g x)=d g_{x}\left(v_{i}\right)$ form a global parallel frame for $\nu(N)$.

(2) The principal curvatures of $N$ with respect to any parallel normal field are constant.

Proof. (0) follows from 3.2(4) and (5), and (1) follows from 3.2(1) and 3.6(ii). Since $G$ acts on $M$ by isometries, $A_{d g_{x}(v)}$ and $A_{v(x)}$ have the same eigenvalues. Then (2) follows from 3.6(ii).

6.2. Definition. Let $N$ be a submanifold of $M$ and $v$ a parallel normal field on $N$. Then the parallel set $N_{v}$ is defined to be $\{\exp (v(x)) \mid x \in N\}$.

If $N$ is compact then $N_{v}$ is, for small $v$, diffeomorphic to $N$. Although in general $N_{v}$ may have singularities, if $N$ is a principal orbit of a Riemannian $G$-manifold with sections then each parallel set is also a $G$-orbit and hence a smooth submanifold (whose dimensions may vary with $v$ ). So the orbit foliation in this case is determined by the Riemannian geometry of a single principal orbit. When $M$ is a space form (i.e., has constant sectional curvature) submanifolds which have properties 6.1(1) and (2) have been studied. We recall

6.3. Definition $[\mathbf{T}]$. A connected compact submanifold $M^{n}$ of a space form $N^{n+k}(c)$ is isoparametric if

(i) $\nu(M)$ is flat,

(ii) the principal curvatures of $M$ with respect to any parallel normal field are constant. 
It follows from 6.1 that we have

6.4. Proposition. Suppose $G$ acts on $N^{n}(c)$ isometrically and admits sections. Then the principal orbits are isoparametric.

A submanifold $M$ of a space form $N$ is called full if $M$ is not contained in any totally geodesic hypersurface of $N$.

6.5. THEOREM. Suppose $M^{n}$ is a full and isoparametric submanifold of $\mathbb{P}^{n+k}$, and $G=\{g \in \mathbf{O}(n+k) \mid g(M) \subset M\}$ acts on $M$ transitively. Then the representation $\rho: G_{0} \rightarrow \mathbf{O}(n+k)$ is polar and $M$ is a principal $G_{0}$-orbit, where $G_{0}$ is the connected component of $G$.

PROOF. We need the following result concerning isoparametric submanifolds proved in $[\mathbf{T}]$ : there exist $p(p \geq k)$ parallel normal fields (the curvature normals) $v_{1}, \ldots, v_{p}$ such that the shape operator $A_{v}$ has eigenvalues $\left\langle v, v_{1}\right\rangle, \ldots,\left\langle v, v_{p}\right\rangle$, and $v_{1}, \ldots, v_{k}$ is a global normal frame field on $M$. Note that $A_{v}$ and $A_{g v}$ have the same eigenvalues, so there is a permutation $\sigma$ of $1, \ldots, p$ such that $\left\langle g v(x), v_{i}(g x)\right\rangle=$ $\left\langle v(x), v_{\sigma(i)}(x)\right\rangle$. Since $\left\langle g v(x), v_{i}(g x)\right\rangle=\left\langle v(x), g^{-1} v_{i}(g x)\right\rangle$ and $v_{1}, \ldots, v_{k}$ is a base of $\nu(M)$, we have $g^{-1} v_{i}(g x)=v_{\sigma(i)}(x)$. Therefore to each curve $g_{t}$ in $G_{0}$ such that $g_{0}=$ id we have associated a continuous map $t \rightarrow \sigma\left(g_{t}\right)$ from $(-\varepsilon, \varepsilon)$ to $S_{p}$ and $\sigma(0)=$ id. Hence $\sigma\left(g_{t}\right) \equiv$ id, which implies that every parallel normal field is $G_{0}$-invariant, so the slice representation of the orbit $M$ is trivial. Thus $M$ is a principal orbit and by 3.6 the principal horizontal distribution $\mathcal{H}$ is integrable. Finally it follows from 3.4 that $\rho$ is polar.

A submanifold $M$ of a Riemannian manifold $N$ is called ambient homogeneous if $M$ is an orbit of an isometric action on $N$. Therefore being isoparametric and ambient homogeneous characterize the principal orbits of polar representations. So it follows from Dadok's Theorem 4.4 that we have

6.6. PROPOSITION. Every ambient homogeneous isoparametric submanifold of $\mathbb{P}^{n}$ arises as a principal orbit of the isotropy representation of a symmetric space.

However there are also many nonhomogeneous isoparametric submanifolds in $\mathbb{B}^{n}$ $[\mathbf{O T}, \mathbf{F K M}]$. It seems that the conditions in 6.1 are stronger than those in 6.3 for isoparametric, but we note that $6.1(0)$ is always true for submanifolds of space forms, and it is also known that if $M$ is isoparametric in $\mathbb{R}^{n}$ then the holonomy of $\nu(M)$ is trivial. Hence isoparametric submanifolds of $\mathbb{P}^{n}$ can be thought as a purely geometric analogue of principal orbits of polar representations. Moreover the parallel foliation of an isoparametric submanifold of $\mathbb{P}^{n}$ is an orbitlike foliation. To be more specific we have

6.7. THEOREM [T, HPT, PT]. Suppose $M^{n}$ is an isoparametric submanifold of $\mathbb{R}^{n+k}$. Then there is a Weyl group $W$ associated to $M$, and

(1) $W$ acts on $\exp \left(\nu(M)_{x}\right)\left(=x+\nu(M)_{x}\right)$ isometrically (=orthogonally) for all $x$ in $M$, and $W$ acts diffeomorphically on $M$,

(2) every parallel set of $M$ is a smooth submanifold (of dimension $\leq n$ ) of $\mathbb{P}^{n+k}$,

(3) let $\Delta$ denote the Weyl chamber of the $W$-space $\exp \left(\nu(M)_{x_{0}}\right)$ which contains $x_{0}$, and let $y$ be in $\Delta$; then there is a unique parallel manifold $M_{v}$ containing $y$ (which we denote also by $M_{y}$ ) and $\bigcup\left\{M_{y} \mid y \in \Delta\right\}=\mathbb{R}^{n+k}$, 
(4) let $\Pi: \mathbb{R}^{n+k} \rightarrow \Delta$ be the canonical projection, $\Pi\left(M_{y}\right)=y$; then $\Pi$ is a quasi-homogeneous stratified Riemannian submersion ( $c f .[\mathbf{P T}])$.

Now it is natural to make the following definition.

6.8. Definition. A submanifold $M$ of a Riemannian manifold $N$ is called isoparametric if

(0) $\exp \left(\nu(M)_{x}\right)$ is a properly embedded totally geodesic submanifold of $N$ for all $x$ in $M$,

(1) $\nu(M)$ is flat and has trivial holonomy,

(2) the principal curvatures of $M$ with respect to any parallel normal field are constant.

It follows from 6.1 that the principal orbits of a complete Riemannian $G$-manifold with sections are isoparametric. However it is not known whether there are analogues of 6.5 and 6.7 for Riemannian symmetric spaces.

\section{REFERENCES}

[BS] R. Bott and H. Samelson, Applications of the theory of Morse to symmetric spaces, Amer. J. Math. 80 (1958), 964-1029.

[B] G. E. Bredon, Introduction to compact transformation groups, Academic Press, New York, 1972.

[C] C. Chevalley, Invariants of finite groups generated by reflections, Amer. J. Math. 77 (1955), 778-782.

[Co1] L. Conlon, Variational completeness and K-transversal domains, J. Differential Geom. 5 (1971), 135-147.

[Co2] _ A class of variationally complete representations, J. Differential Geom. 7 (1972), 149-160.

Da1 J. Dadok, On the $C^{\infty}$ Chevalley theorem, Adv. in Math. 44 (1982), 121-131.

[Da2] _ Polar coordinates induced by actions of compact Lie groups, Trans. Amer. Math. Soc. 288 (1985), 125-137.

[D] M. Davis, Smooth G-manifolds as collections of fiber bundles, Pacific J. Math. 77 (1978), 315-363

[FKM] D. Ferus, H. Karcher and H. F. Münzner, Cliffordalgebren und neue isoparametrische hyperflächen, Math. Z. 177 (1981), 479-502.

[H1] R. Hermann, Variational completeness for compact symmetric spaces, Proc. Amer. Math. Soc. 11 (1960), 544-546.

[H2] _ Totally geodesic orbits of groups of isometries, Nederl. Acad. Wetensch. Proc. Ser. A 65 (1962), 291-298.

[HHS ] W. T. Hsiang, W. Y. Hsiang and I. Sterling, On the construction of codimension two minimal immersions of exotic spheres into Euclidean spheres, preprint.

[Hs1 $]$ W. Y. Hsiang, On the compact homogeneous minimal submanifolds, Proc. Nat. Acad. Sci. U.S.A. 56 (1966), 56.

[Hs2]_, Minimal cones and the spherical Berstein problem. I, Ann. of Math. (2) 118 (1983), 61-73; II, Invent. Math. 74 (1983), 351-369.

[HL] W. Y. Hsiang and B. H. Lawson, Jr., Minimal submanifolds of low cohomogeneity, J. Differential Geom. 5 (1971), 1-38.

[HPT $]$ W. Y. Hsiang, R. S. Palais and C. L. Terng, The topology and geometry of isoparametric submanifolds of Euclidean spaces, announcement, Proc. Nat. Acad. Sci. U.S.A. 82 (1985), 4863-4865; full paper in preparation.

[KN] S. Kobayashi and K. Nomizu, Foundations of differential geometry, Interscience, New York, 1969.

[M] H. F. Münzner, Isoparametric Hyperflächen in Sphären. I, Math. Ann. 251 (1980), 57-71; II, Math. Ann. 256 (1981), 215-232.

[O] B. O'Neill, The fundamental equations of a submersion, Michigan Math. J. 13 (1966), 459469. 
[OT] H. Ozeki and M. Takeuchi, On some types of isoparametric hypersurfaces in spheres. 1, 2, Tôhoku Math. J. 27 (1975), 515-559; 28 (1976), 7-55.

[P] R. S. Palais, On the existence of slices for actions of non-compact Lie groups, Ann. of Math. (2) 73 (1961), 295-323.

[PT] R. S. Palais and C. L. Terng, Reduction of variables for minimal submanifolds, Proc. Amer. Math. Soc. 98 (1986), 480-484.

[S1] G. Schwarz, Smooth functions invariant under the action of a compact Lie group, Topology 14 (1975), 63-68.

[S2] _ Lifting smooth homotopies of orbit spaces, Inst. Hautes Études Sci. Publ. Math. 51 (1980), 37-135.

[Sz1] J. Szenthe, A generalization of the Weyl group, Acta Math. Hungar. 41 (1983), 347-357.

[Sz2] _ Orthogonally transversal submanifolds and the generalizations of the Weyl group, Period. Math. Hungar. 15 (1984), 281-299.

[T] C. L. Terng, Isoparametric submanifolds and their Coxeter groups, J. Differential Geom. 21 (1985), 79-107.

Department of Mathematics, Brandeis University, Waltham, MassachuSETTS 02254

Department of Mathematics, Northeastern University, Boston, MassachuSETTS 02115 